\title{
Editorial
}

\section{Somos los traumatólogos inmunes al Burnout?}

\author{
Mario Orrego ${ }^{1}$ María Jesús Lira ${ }^{1}$ Sebastián Irarrázaval ${ }^{1}$ \\ 1 Pontificia Universidad Catòlica de Chile
}

Rev Chil Ortop Traumatol 2020;61:75.

El agotamiento ocupacional, o más comúnmente denominado "burnout", es un síndrome en el cual la persona experimenta simultáneamente agotamiento emocional, despersonalización y una percepción de falta de realización personal. ${ }^{1}$ Tradicionalmente se ha asociado a aquellas ocupaciones o profesiones en donde se ejerce un rol de cuidado, siendo los médicos una población de riesgo debido a los altos niveles de estrés y exigencias a los que se ven expuestos. ${ }^{2}$

En personas con burnout se ha descrito un mayor número de errores, disminución en la calidad del cuidado otorgado a los pacientes, así como una menor productividad, generando una sobrecarga en los sistemas de salud y deterioro de la relación médico-paciente. ${ }^{3,4}$ En el área de Ortopedia y Traumatología, se han reportado tasas entre un 40 y $70 \%$ de agotamiento ocupacional, variando según el grado de formación y desarrollo profesional, ${ }^{4,5}$ desconociéndose cifras nacionales.

Comprender en mayor medida éste fenómeno a nivel local cobra aún más importancia en la realidad nacional, donde los especialistas se ven enfrentados a nuevos estresores en consecuencia de la crisis social y actual pandemia, pudiendo estar aún más expuestos a experimentar burnout. ${ }^{6}$

Es así como comenzamos este número con la presentación del artículo del grupo de la Facultad de Medicina Clínica Alemana-Universidad del Desarrollo, "Burnout y calidad de vida en traumatólogos chilenos: ¿Estamos realmente bien?", donde los autores muestran un preocupante porcen- taje de agotamiento ocupacional en la mitad de los especialistas encuestados, afectando predominantemente a jóvenes que atienden en el sistema de salud chileno.

En próximas ediciones, esperamos recibir estudios que nos permitan seguir ahondando en este tema, con el fin de internalizar y contribuir al bienestar emocional de nuestros profesionales, especialistas y residentes a nivel nacional.

\section{Referencias}

1 Maslach C, Schaufeli WB, Leiter MP. Job burnout. Annu Rev Psychol 2001;52:397-422

2 Shanafelt TD, Boone S, Tan L, et al. Burnout and satisfaction with work-life balance among US physicians relative to the general US population. Arch Intern Med 2012;172(18):1377-1385

3 West CP, Dyrbye LN, Shanafelt TD. Physician burnout: contributors, consequences and solutions. J Intern Med 2018;283(06): 516-529

4 Sargent MC, Sotile W, Sotile MO, Rubash H, Barrack RL. Quality of life during orthopaedic training and academic practice. Part 1: orthopaedic surgery residents and faculty. J Bone Joint Surg Am 2009;91(10):2395-2405

5 Travers V. Burnout in orthopedic surgeons. Orthop Traumatol Surg Res 2020;106(1S):S7-S12. Doi: 10.1016/j.otsr.2019.04.029 [Internet]

6 Morgantini LA, Naha U, Wang H, Francavilla S, Acar Ö, Flores JM, Crivellaro S, Moreira D, Abern M, Eklund M, Vigneswaran HT WS. Factors Contributing to Healthcare Professional Burnout During the COVID-19 Pandemic: A Rapid Turnaround Global Survey. 2020;1(617):1-17
Address for correspondence Mario Orrego, Pontificia Universidad Catòlica de Chile, Chile

(e-mail: mario.orrego@gmail.com).
DOI https://doi.org/ 10.1055/s-0040-1721025. ISSN 0716-4548.
Copyright $\odot 2020$ by Thieme Revinter Publicações Ltda, Rio de Janeiro, Brazil
License terms

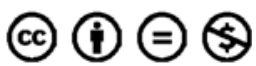

